\title{
Enfermera-matrona: beneficios, competencias e intervenciones
}

\section{Nurse-midwife: benefits, abilities and interventions}

\section{Enfermeira obstétrica: benefícios, competências e intervenções}

\author{
Diana Camila Berruecos-Prada ${ }^{1}$ \\ Esmeralda María Calderón-Rivas ${ }^{2}$ \\ Karen Sofía Rodríguez-Ramírez ${ }^{3}$ \\ Mary Julieth Guayacundo-Aldana ${ }^{4}$ \\ Ruby Magdaly Mancera-García ${ }^{5}$ \\ Ruth Alexandra Castiblanco-Montañez ${ }^{6}$
}

\section{Resumen}

Introducción: La enfermera permite a las gestantes tener autonomía en el embarazo y parto, pero no es una profesión reconocida actualmente; sin embargo, la Resolución 3280 de 2018 define los lineamientos técnicos y operativos de la Ruta Integral de Atención en Salud para la Población Materno Perinatal y establece las directrices para su operación, destacando la participación del profesional de la enfermería en la consulta preconcepcional y controles prenatales. Objetivo: Reconocer los beneficios de la atención por parte de las enfermeras durante la gestación y el trabajo de parto reportados en la literatura. Metodología: Revisión integrativa de la literatura con un período de búsqueda de febrero-mayo del 2020 en EBSCO, Pubmed, LILACS, Cuiden y Google académico con los siguientes términos DeCS-MeSH: Matronas, complicaciones, parto, embarazo, cuidados de enfermería y puerperio, operadores booleanos AND-OR. Se obtuvieron 44.966 artículos en inglés, español y portugués agrupados según su título, resumen, texto completo, nivel de evidencia y grado de recomendación; no obstante, se seleccionaron 33 artículos para realizar la lectura crítica. Se debe aclarar que cumplieron las consideraciones éticas para estudios documentales. Resultados: A través de un análisis de contenido se construyeron 4 temáticas: beneficios de la asistencia gestacional y de parto por la enfermera, competencias e intervenciones de la enfermera en la atención integral de la mujer gestante, consideraciones de riesgo en la asistencia de la gestación y el parto por enfermeras y retos de la enfermera en la atención durante el embarazo, parto y puerperio. Conclusión: La asistencia de la enfermera en gestaciones de bajo riesgo aporta múltiples beneficios a la mujer, reduce el tiempo de nacimiento y el dolor; además posibilita la identificación de riesgos de manera oportuna.

Palabras clave: Cuidados de Enfermería, complicaciones del trabajo de parto, puerperio, Matronas.

\footnotetext{
Abstract

Introduction: Nurse-midwifery allows expectant mothers to have autonomy during their pregnancy and birth, but it is not currently recognized as a profession; however, the legal resolution 3280 of 2018 defines the technical and operational guidelines of the Comprehensive Health Care Pathways for the Maternal and Perinatal Health Population and establishes their operational guidelines, emphasizing the participation of the nursing professional in the preconception consultation and prenatal care. Objective: Recognize the benefits of the care provided by nurses during the pregnancy and labor reported in the literature. Methodology: Comprehensive review of the literature with a searching period between February-May
}

\begin{abstract}
Autor de correspondencia*
${ }^{1 *}$ Estudiante de VI semestre. Semillero de Investigación, Red de Cuidado, Ciencia y Arte de Enfermería. Fundación Universitaria de Ciencias de Salud - FUCS. Bogotá, Colombia. Correo: dcberruecos@fucsalud.edu.co (1D) 0000-0002-7668-773X
\end{abstract}

${ }^{2}$ Estudiante de VI semestre. Semillero de Investigación, Red de Cuidado, Ciencia y Arte de Enfermería. Fundación Universitaria de Ciencias de Salud - FUCS. Bogotá, Colombia. Correo: ecalderon@fucsalud.edu.co (1) 0000-0003-3960-4533

${ }^{3}$ Estudiante de VI semestre. Semillero de Investigación, Red de Cuidado, Ciencia y Arte de Enfermería. Fundación Universitaria de Ciencias de Salud - FUCS. Bogotá, Colombia. Correo: ksrodriguez3@fucsalud.edu.co (1) 0000-0003-3733-0321

${ }^{4}$ Estudiante de VI semestre. Semillero de Investigación, Red de Cuidado, Ciencia y Arte de Enfermería. Fundación Universitaria de Ciencias de Salud - FUCS. Bogotá, Colombia. Correo: miguayacundo@fucsalud.edu. co (다 0000-0002-4163-5024

${ }^{5}$ Estudiante de VI semestre. Semillero de Investigación, Red de Cuidado, Ciencia y Arte de Enfermería. Fundación Universitaria de Ciencias de Salud - FUCS. Bogotá, Colombia. Correo: rmmancera@fucsalud.edu.co (D) 0000-0001-7668-7126

${ }^{6}$ Enfermera. Magister en Salud Pública. Profesor asistente. Coordinadora de Investigaciones. Grupo Perspectivas del Cuidado. Fundación Universitaria de Ciencias de la Salud - FUCS. Bogotá, Colombia. Correo: racastiblanco@ fucsalud.edu.co (D) 0000-0001-8328$\underline{9601}$ 


\section{Scientific Journal of Nursing}

of 2020 in the EBSCO, Pubmed, LILACS, Cuiden and Google Scholar databases with the following DeCS-MeSH terms: Midwifes, complications, labor, pregnancy, nursing care and postpartum period, logical connectors AND-OR. 44.966 papers in English, Spanish and Portuguese were obtained and grouped according to their title, abstract, full text, level of evidence and level of recommendation; however, 33 of these papers were chosen for critical reading. It should be made clear that the ethical considerations were met for documentary research. Results: Through the analysis of the content 4 topics were built: benefits of prenatal care and assisted delivery, abilities and interventions of the nurse for the comprehensive care of the expectant mother, risk considerations of the nurse during prenatal care and delivery and nursing care challenges during the pregnancy, labor and postpartum period. Conclusion: The assistance of the nurse during low-risk pregnancies provides multiple benefits for the woman, reduces the pain and period of labor; also enabling the identification of risks in a timely manner.

Keywords: Nursing care, complications during labor, postpartum period, midwifes.

\section{Resumo}

Introdução: a enfermeira permite às gestantes ter autonomia na gravidez e o parto, porém é uma profissão não reconhecida atualmente, entretanto, a Resolução 3280 de 2018 define os lineamentos técnicos e operativos da Rota de Atendimento Integral à Saúde para a população Materna e Perinatal e estabelece as diretrizes para a sua operação, destacando a participação do profissional de enfermagem no atendimento pré-natal. Objetivo: Reconhecer os benefícios da atenção por parte de enfermeiras durante a gestação e o trabalho de parto referidos na literatura. Materiais e Métodos: Revisão integrativa da literatura realizada no período de fevereiro -maio de 2020 em EBSCO, Pubmed, LILACS, Cuidem e Google Scholar empregando os termos DeCS-MeSH: Enfermeiras obstétricas, complicações, parto, gravidez, cuidados de enfermagem e período pós-parto, operadores AND-OR. Obtiveram-se 44.966 artículos em inglês, espanhol e português agrupados segundo o título, resumo, texto completo, nível de evidência e grado de recomendação; entretanto, se selecionaram 33 artículos para realizar a leitura crítica. Deve-se esclarecer que as considerações éticas para estudos documentais. $\boldsymbol{R} \boldsymbol{e}$ sultados: Através da analise de conteúdo construíram-se 4 temáticas: benefícios de assistência gestacional e de parto pela enfermeira, competências e intervenções da enfermeira no atendimento integral da mulher grávida, considerações de risco na assistência da gestação e o parto por enfermeiras e desafios da enfermeira no atendimento durante a gravidez o parto e pós-parto. Conclusão: A assistência da enfermeira em gestações de baixo risco contribui com múltiplos beneficios à mulher, reduze o tempo do nascimento e a dor; além disso possibilita a identificação de riscos da maneira oportuna.

Palavras-chave: Cuidados de Enfermagem, complicações do trabalho de parto, pós-parto, enfermeira obstétrica.
Recibido: 18 diciembre 2020 Aprobado: 12 abril 2021

Para citar este artículo / To reference this article / Para citar este artigo:

Berruecos-Prada DC, Calderón-Rivas EM, Rodríguez-Ramírez KS, Guayacundo-Aldana MJ, Mancera-García RM, Castiblanco-Montañez RA. Enfermera-matrona: beneficios, competencias e intervenciones. Rev. cienc. cuidad. 2021; 18(2):96-110. https://doi. org $/ 10.22463 / 17949831.2633$

(C) Universidad Francisco de Paula Santander. Este es un artículo bajo la licencia CC-BY-NC-ND

\section{Introducción}

Culturalmente se ha considerado el nacimiento como un evento normal y natural, basado en creencias religiosas donde el embarazo se califica como un hecho divino; asimismo, el parto es el proceso final del embarazo y el inicio de la vida del neonato, donde no sólo se ven implicadas las prácticas médicas, sino también el entorno psicosocial de la mujer (1-2).

Se debe precisar que las complicaciones están relacionadas con el desarrollo de las condiciones que ponen en riesgo la vida de la gestante o del producto de la gestación, tales como: preeclampsias severas, eclampsias, hemorragias obstétricas severas, sepsis, entre otras. Una de las principales complicaciones durante el parto es la muerte materna en un $75 \%$ de los casos, derivada en primer lugar por hemorragia obstétrica (17\%), seguida por el trastorno hipertensivo del embarazo con el $14,7 \%$, la sepsis obstétrica $9,3 \%$ y eventos tromboembólicos con un 3,6\%(3). El shock hemorrágico es otro de los trastornos más frecuentes de morbi-mortalidad materna, causando la muerte a 1 de cada 130 mujeres; sin embargo, se debe tener presente que este 
es un desenlace negativo que puede ocurrir durante el embarazo, el parto o postparto. También es frecuente el sufrimiento fetal en más del $60 \%$ y está fuertemente asociado a un pobre seguimiento durante el puerperio inmediato (4).

Existen tres tipos de modelos de atención durante el parto: el primero, asistido por las enfermeras para gestantes con estado de salud estable, son embarazos de bajo riesgo y/o que requieran intervenciones mínimas en el procedimiento; el segundo, se refiere a la atención clínica obstétrica; $y$, el tercero conforma la atención mixta de enfermera y obstetra (5). A nivel mundial, las mujeres que residen en zonas urbanas acceden a la atención con parteras en un $81 \%$, en comparación con las que habitan en las zonas rurales $(49 \%)$, siendo esta última población la que cuenta con características que aumentan su vulnerabilidad, especialmente durante la gestación a causa de sus DDS, por lo que es allí donde se observan más casos de muertes maternas (6).

Por su parte, la Organización Mundial de la Salud promueve estrategias que fortalecen la calidad de la atención en enfermería y partería por medio de los cuidados especializados, así como con la participación y desarrollo de políticas públicas sanitarias. Estas actividades permiten que el personal de salud ejerza un papel fundamental a futuro en la salud de pacientes y comunidades; es decir, que se facilita el empoderamiento del personal de enfermería, no solamente en los profesionales, sino también en técnicos, en la atención de los partos de bajo riesgo; es decir, que se pretende cumplir con los Objetivos de Desarrollo Sostenible, específicamente el numeral 3 , con el cual se busca reducir la mortalidad materno-infantil. (7-10)

Conviene subrayar que en algunos países este rol lo desempeña una enfermera de práctica avanzada que es experta, con habilidades para la toma de decisiones clínicas complejas y con las suficientes competencias para ejercer un papel extendido. Es por eso, que después del pregrado, realiza estudios de especialización, maestrías y/o doctorados, reconociéndoles como matronas. Dependiendo del país en el que se realice, esta especialización tendrá una duración de 1 a 4 años. De ahí, que los servicios que presta la matrona incluyen medidas preventivas, la promoción del parto normal, detección de complicaciones en la madre y el niño, acceso a la atención médica u otra asistencia apropiada y la atención de medidas de emergencia (11-14).

En Colombia actualmente esta figura de matrona, no se ha hecho efectiva en la prestación de servicios de salud, aún a pesar de la presencia de antecedentes significativos como: Funcionamiento de la primera escuela de Comadronas y Enfermeras de 1920-1937; formación de especialistas en enfermería obstétrica por la Universidad del Valle en la década del 70; y, de enfermeras materno perinatales por la Universidad Nacional desde 1983. Por otra parte, se cuenta con la Resolución 412 de 2000 y la estrategia IAMI, en donde se establece el acompañamiento por parte del profesional de enfermería desde la consulta prenatal hasta el puerperio, así como la vinculación de un familiar o persona cercana durante el mismo. Por otro lado, la Resolución 3280 de 2018 define los lineamientos técnicos y operativos de la Ruta Integral de Atención para la Promoción y Mantenimiento de la Salud y la Ruta Integral de Atención en Salud para la Población Materno Perinatal estableciendo las directrices para su operación, entre las que se destacan: la participación del profesional de enfermería no sólo en la consulta de la preconcepción y controles prenatales, sino que también tiene facultad para atender partos de baja complejidad (15).

Actualmente no se tiene acompañamiento de la enfermera antes, durante o después del parto, ya sea por desconocimiento de las gestantes o porque las instituciones de salud no lo permiten, aunque está claramente contemplado en los lineamientos de la Ruta Integral de la Atención materno perinatal, en donde se indica que la mujer en trabajo de parto debe estar acompañada, a fin de que reciba todas las atenciones e intervenciones necesarias oportunamente; igualmente, se le debe brindar apoyo físico, psicológico y espiritual de manera individual y de forma continua, siempre en función de la persona que ella elija, aunque esto puede no llevarse a cabo (16). Lo anterior es una limitante importante para el ejercicio en Colombia de las enfermeras especialistas en obstetricia en el ámbito intra o extrahospitalario. Desde este punto de vista es muy importante que estas intervenciones de cuidado se ejerzan teniendo en cuenta: los diferentes contextos y sus tradiciones culturales como por ejemplo en las comunidades afrodescendientes, indígenas o campesinas. Al respecto se sabe que en los países desarrollados el acompañamiento a la mujer durante el embarazo, parto, puerperio y atención al recién nacido por enfermeras certificadas, es una práctica común en hospitales y domicilios (15).

En definitiva con este artículo, se tiene como objetivo describir los beneficios de la atención de las enfermeras durante la gestación y el trabajo de parto reportados en la literatura.

\section{Metodología}

Se realizó una investigación secundaria, partiendo del análisis de pruebas disponibles originadas en una 
fuente primaria, con el objetivo de responder a la pregunta (17). Se utilizó un diseño de revisión integrativa (18), en seis fases. En la primera se identificó el problema y se planteó la pregunta con el modelo "PICO" ¿En madres gestantes cuáles son los beneficios de la atención de las enfermeras en el parto?". Posteriormente, se efectuó una búsqueda sistemática, utilizando los descriptores DeCS)/términos MeSH junto con los operadores booleanos por medio de la ecuación ("Matronas" OR "Comadrona" OR "Matronería" OR "Partera") AND ("Complicaciones") AND ("Parto" OR "embarazo" OR "gestación" OR "Atención tradicional del nacimiento"), en varias bases de datos, interfaces electrónicas y metabuscadores, tales como: EBSCO/ Medline, Pubmed/Medline, LILACS, Cuiden y Google Académico, para obtener mayores resultados.

En la segunda fase, se establecieron como criterios de inclusión: artículos en español, inglés o portugués publicados entre 2015 y 2020, con una fuerte relación o similitud a la temática objeto de estudio. En la tercera fase se categorizaron los artículos seleccionados en Excel de acuerdo a su título, resumen, texto completo, nivel de evidencia y grado de recomendación. Al respecto se tuvieron en cuenta los modelos encontrados en CASPe y la escala Strobe para los diseños de corte transversal, casos y controles y observacional. En la cuarta fase se procedió a realizar lectura crítica de los mismos (19-20).

Como quinta fase, se efectuó la clasificación de los resultados obtenidos en 3 temáticas con el fin de clasificar con mayor facilidad la información obtenida. En la sexta fase, con la información que se obtuvo se elaboró el resumen del artículo.

Las consideraciones éticas que se tuvieron en cuenta fueron: La Resolución 8430 de 1993 que establece las normas científicas, técnicas y administrativas para la investigación en salud; el artículo 29 de la ley 911 de 2004 en donde se resalta como obligación del investigador, el salvaguardar la dignidad y la integridad de los seres humanos durante cualquier investigación para la evolución médica (21-22). Y, por último, la ley 1915 de 2018 por la cual se dictan las disposiciones sobre derechos de autor (23).

\section{Análisis}

La búsqueda inicial dio como resultado un total de 44.966 artículos y según los criterios establecidos se encontraron 33 publicados en el periodo de 2015 2020, en países como Estados Unidos, España y Brasil; de los cuales: 25 están en inglés, 17 en español y 3 en portugués. Respecto al tipo de investigación: 22 son de tipo primaria y 12 de tipo secundaria. De acuerdo al enfoque: 20 estudios eran cualitativos y 14 de tipo cuantitativo. Según su diseño: 13 son de corte transversal, 13 de tipo integrativo, 1 ensayo clínico, 2 de casos y controles, 8 de serie de casos y 1 reporte de caso. Ver Diagrama 1.

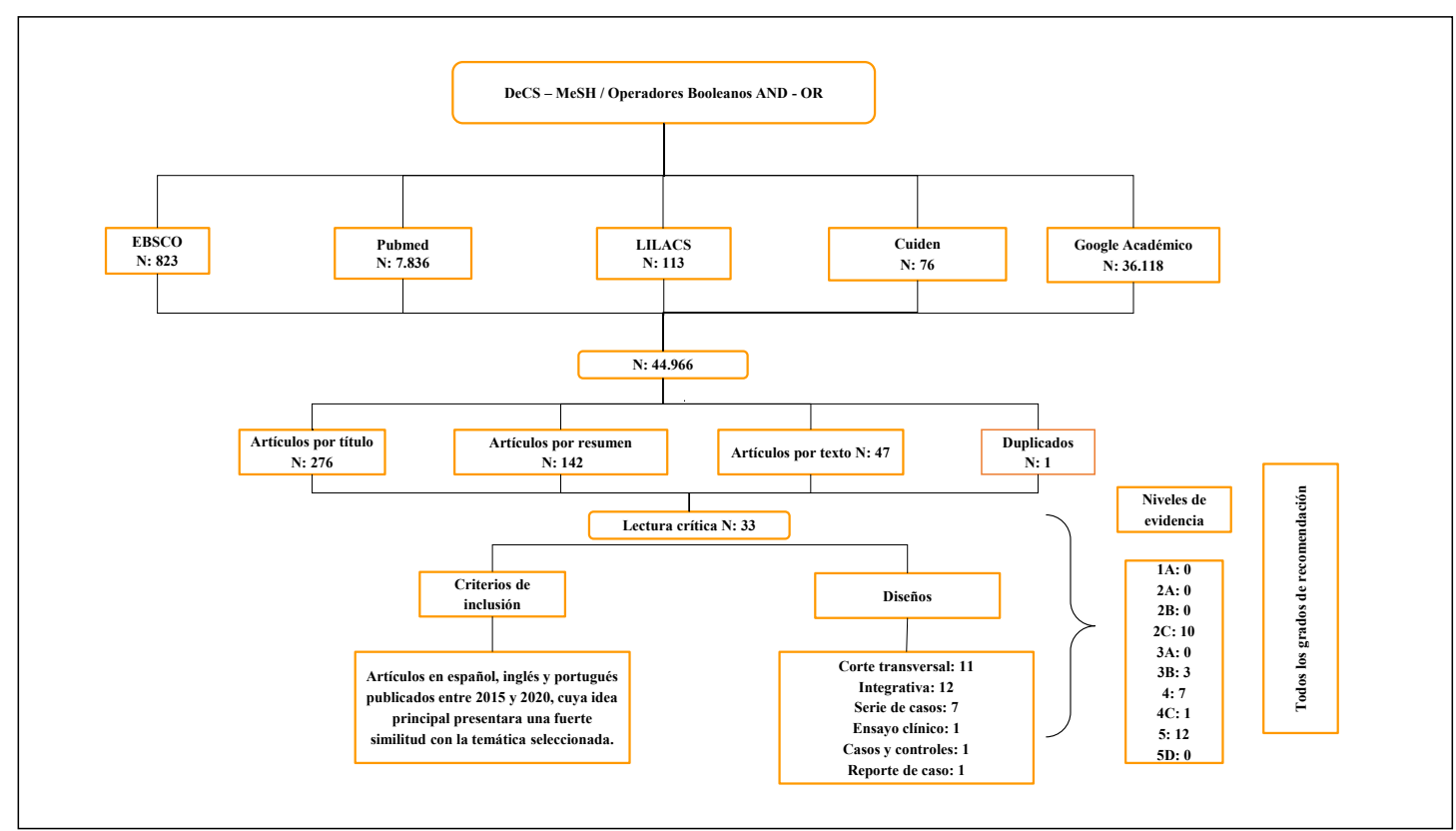

Diagrama 1: Árbol de proceso de búsqueda y selección de artículos.

Fuente: las autoras, 2020. 
A estos artículos se les aplicó las parrillas de lectura crítica según el diseño metodológico, clasificándolos según el nivel de evidencia: 10 con nivel $2 \mathrm{C}, 3$ con el nivel 3B, 7 con el nivel 4, 1 con el nivel 4 C y 12 con nivel 5. Finalmente, respecto a los grados de recomen- dación: 13 con grado B, 6 de grado $\mathrm{C}$ y 13 de grado D, tal y como se ve reflejado en la Tabla 1.

Tabla 1. Síntesis de evidencias

\begin{tabular}{|c|c|c|c|c|}
\hline Nombre Del Título & Año & Diseño & N.E & G.R \\
\hline $\begin{array}{c}\text { Panorámica internacional en relación a las recomen- } \\
\text { daciones, práctica clínica y legislación del parto en } \\
\text { casa }(24) \text {. }\end{array}$ & 2017 & Integrativo & 5 & $\begin{array}{l}\text { D, Ni se recomienda ni se } \\
\text { desaprueba }\end{array}$ \\
\hline $\begin{array}{l}\text { Historia de las enfermeras obstétricas: importancia } \\
\text { de sus cuidados en la atención al parto }(25) \text {. }\end{array}$ & 2018 & Integrativo & 5 & $\begin{array}{l}\mathrm{D}, \text { Ni se recomienda ni se } \\
\text { desaprueba }\end{array}$ \\
\hline $\begin{array}{l}\text { A vivência de adolescentes assistidas por enfer- } \\
\text { meiros obstetras durante o processo de parturição } \\
\text { (26). }\end{array}$ & 2017 & $\begin{array}{l}\text { Descriptivo/ Serie } \\
\text { de casos }\end{array}$ & 4 & $\begin{array}{l}\text { C, Recomendación favor- } \\
\text { able pero no concluyente }\end{array}$ \\
\hline $\begin{array}{l}\text { El parto humanizado como necesidad para la } \\
\text { atención integral a la mujer (27). }\end{array}$ & 2018 & Integrativo & 5 & $\begin{array}{l}\text { D, Ni se recomienda ni se } \\
\text { desaprueba }\end{array}$ \\
\hline $\begin{array}{l}\text { Nurses' and midwives' experiences of providing } \\
\text { group antenatal and postnatal care at } 18 \text { health cen- } \\
\text { ters in Rwanda: A mixed methods study }(28) \text {. }\end{array}$ & 2019 & Integrativo & 5 & $\begin{array}{l}\text { D, Ni se recomienda ni se } \\
\text { desaprueba }\end{array}$ \\
\hline $\begin{array}{l}\text { Factors affecting midwives' confidence in intrapar- } \\
\text { tum care: A phenomenological study (29). }\end{array}$ & 2015 & $\begin{array}{l}\text { Descriptivo / Serie } \\
\text { de casos }\end{array}$ & $4 \mathrm{C}$ & $\begin{array}{l}\text { C, Recomendación favor- } \\
\text { able, pero no concluyente }\end{array}$ \\
\hline El proceso del parto en el domicilio (30). & 2018 & Integrativo & 5 & $\begin{array}{l}\mathrm{D}, \mathrm{Ni} \text { se recomienda ni se } \\
\text { desaprueba }\end{array}$ \\
\hline Aspectos bioéticos del parto natural (31). & 2015 & $\begin{array}{l}\text { Descriptivo/ Corte } \\
\text { transversal }\end{array}$ & $2 \mathrm{C}$ & $\begin{array}{l}\text { B, Recomendación favor- } \\
\text { able }\end{array}$ \\
\hline $\begin{array}{l}\text { Práticas obstétricas de uma parteira: contribuições } \\
\text { para a gestão do cuidado de enfermagem à parturi- } \\
\text { ente (32). }\end{array}$ & 2019 & $\begin{array}{l}\text { Descriptivo, reporte } \\
\text { de caso. }\end{array}$ & $3 \mathrm{~B}$ & $\begin{array}{l}\text { B, Recomendación favor- } \\
\text { able }\end{array}$ \\
\hline $\begin{array}{l}\text { Progresses and challenges of utilizing traditional } \\
\text { birth attendants in maternal and child health in Nige- } \\
\text { ria (33). }\end{array}$ & 2017 & Integrativo & 5 & $\begin{array}{l}\mathrm{D}, \mathrm{Ni} \text { se recomienda ni se } \\
\text { desaprueba }\end{array}$ \\
\hline $\begin{array}{l}\text { Support provided by midwives to women during } \\
\text { labour in a public hospital, Limpopo Province, South } \\
\text { Africa: a participant observation study (34). }\end{array}$ & 2018 & $\begin{array}{l}\text { Descriptivo/ Serie } \\
\text { de casos }\end{array}$ & 4 & $\begin{array}{l}\mathrm{C} \text {, Recomendación favor- } \\
\text { able pero no concluyente }\end{array}$ \\
\hline $\begin{array}{l}\text { Contribuição da enfermeira obstétrica nas boas práti- } \\
\text { cas da assistência ao parto e nascimento (35). }\end{array}$ & 2018 & $\begin{array}{l}\text { Descriptivo/ Corte } \\
\text { transversal }\end{array}$ & $3 \mathrm{~B}$ & $\begin{array}{l}\text { B, Recomendación favor- } \\
\text { able }\end{array}$ \\
\hline $\begin{array}{l}\text { Labor and birth care by nurse with midwifery skills } \\
\text { in Brazil (36). }\end{array}$ & 2016 & $\begin{array}{l}\text { Descriptivo/ Corte } \\
\text { transversal }\end{array}$ & $2 \mathrm{C}$ & $\begin{array}{l}\text { B, Recomendación favor- } \\
\text { able }\end{array}$ \\
\hline $\begin{array}{l}\text { POPPIE: protocol for a randomised controlled pilot } \\
\text { trial of continuity of midwifery care for women at } \\
\text { increased risk of preterm birth }(37) \text {. }\end{array}$ & 2019 & $\begin{array}{l}\text { Intervención, en- } \\
\text { sayo clínico contro- } \\
\text { lado }\end{array}$ & $2 \mathrm{C}$ & $\begin{array}{l}\mathrm{B}, \text { Recomendación favor- } \\
\text { able }\end{array}$ \\
\hline $\begin{array}{l}\text { Factors influencing the clinical decision-making of } \\
\text { midwives: a qualitative study (38). }\end{array}$ & 2017 & $\begin{array}{l}\text { Descriptivo/ Serie } \\
\text { de casos }\end{array}$ & 4 & $\begin{array}{l}\text { C, Recomendación favor- } \\
\text { able pero no concluyente }\end{array}$ \\
\hline Importancia y beneficios del parto humanizado (39). & 2018 & Integrativo & 5 & $\begin{array}{l}\mathrm{D}, \text { Ni se recomienda ni se } \\
\text { desaprueba }\end{array}$ \\
\hline $\begin{array}{l}\text { Continuity of midwifery carer moderates the effects } \\
\text { of prenatal maternal stress on postnatal maternal } \\
\text { wellbeing: the Queensland flood study (40). }\end{array}$ & 2017 & $\begin{array}{l}\text { Descriptivo/ Serie } \\
\text { de Casos }\end{array}$ & 4 & $\begin{array}{l}\text { C, Recomendación favor- } \\
\text { able, pero no concluyente }\end{array}$ \\
\hline
\end{tabular}




\section{Scientific Journal of Nursing}

Student midwives' awareness, knowledge, and experiences of antenatal anxiety within clinical practice (41).

Relación entre la atención exclusiva de la matrona al puerperio de bajo riesgo y el número de mujeres que acuden a la urgencia de obstetricia por complicaciones posparto (42).

Percepción de embarazadas y matronas acerca de los consejos nutricionales durante la gestación (43).

El acompañamiento por enfermería en la atención del trabajo de parto y parto: una revisión narrativa de la literatura (44).

Midwifery care and patient-provider communication in maternity decisions in the United States (45).

"The midwife helped me ... otherwise I could have died": women's experience of professional midwifery services in rural Afghanistan - a qualitative study in the provinces Kunar and Laghman (46).

Continuity of care by a primary midwife (caseload midwifery) increases women's satisfaction with antenatal, intrapartum and postpartum care: results from the COSMOS randomised controlled trial (47).

Supporting breastfeeding women from the perspective of the midwife: a systematic review of the literature (48).

Factors that influence the provision of intrapartum and postnatal care by skilled birth attendants in lowand middle-income countries: a qualitative evidence synthesis (49).

Midwifery empowerment: National surveys of midwives from Australia, New Zealand and Sweden (50).

Importancia de la asistencia de la matrona durante el puerperio para mejorar la adaptación a la maternidad (51).

Evaluation of competency and skills of skilled birth attendants in Madhya Pradesh, central India (52).

Midwives' oral health recommendations for pregnant women, infants and young children: results of a nationwide survey in Germany (53).

Proceso De Atención De Enfermería En Gestantes Con Complicaciones Obstétricas Por Ruptura Prematura De Membranas (54).

La bienvenida de Leo. Parto en casa (55).

Challenges to the improvement of obstetric care in maternity hospitals of a large Brazilian city: an exploratory qualitative approach on contextual issues (56).
2017

Descriptivo/ Corte transversal

$2 \mathrm{C}$

B. Recomendación favorable

2016

Descriptivo/ Corte transversal

$2 \mathrm{C}$

B, Recomendación favorable

2016

2018

2015

Observacional, analítico: Casos y controles

2020 Observacional, descriptivo: Serie de casos

2016

Descriptivo/ Serie de casos

2016

2017

Integrativo

Integrativo

5

5

D. Ni se recomienda ni se desaprueba

2016

Descriptivo / Corte transversal

2015

Descriptivo / Corte transversal

2018

2016

2018

Descriptivo / Corte transversal

Descriptivo / Corte transversal

Integrativo

2017

2018

Integrativo transversal
5

2B

$2 \mathrm{C}$

B, Recomendación favorable

2C

B, Recomendación favorable

$2 \mathrm{C}$

$\mathrm{B}$, Recomendación favorable

$2 \mathrm{C}$

B, Recomendación favorable

D. Ni se recomienda ni se desaprueba

D, Ni se recomienda ni se desaprueba

$\mathrm{B}$, Recomendación favorable

Fuente: las autoras, 2020. 
Temática 1: Beneficios de la asistencia por la enfermera en la etapa gestacional y de parto

La enfermera está en condiciones de poder cumplir el deseo que tiene la gestante sobre el lugar donde quiere dar a luz, ya sea en el hospital o en su propio domicilio (29). La decisión debe ser tomada únicamente por la madre y sus familiares, teniendo en cuenta que en el hogar se da una atención más centrada en la mujer, ya que ella se convierte en un sujeto activo, teniendo control sobre su cuerpo.

Cabe resaltar que la presencia de la enfermera de práctica avanzada durante el parto tranquiliza de forma significativa a la madre, al considerarla como una persona de confianza que se acopla a su cultura y a sus normas; además, mantiene su confort durante el proceso (29). En este contexto, la enfermera busca garantizar la autonomía de la mujer al momento del parto y privilegia la voluntad de la mujer que va a dar a luz respetando sus tiempos fisiológicos personales; además se tiene presente el derecho a la diversidad, la libre expresión, la libertad de comportamiento de los individuos y grupos (28-30). En este caso, la enfermera es una profesional que está capacitada para brindarle cuidados a la mujer en todos los aspectos, incluyendo el psicosocial, haciendo referencia a sus pensamientos, sentimientos y preparación para el proceso; también tiene en cuenta el aspecto socioeconómico, la red de apoyo, su entorno diario y demás factores que influyen sobre el bienestar de la gestante (39-40).

Por otra parte, se debe considerar que el parto es un evento psico-somático por excelencia, que involucra el cuerpo y la mente de la madre; cuando la enfermera está en el proceso del parto ayuda a disipar miedos y dudas, infunde seguridad, proporciona información amplia y detallada, atiende el bienestar físico y emocional, muestra comprensión, apoyo e intimidad, además de permitir la presencia y participación del padre (27). Todos estos factores, ayudan a crear un entorno de tranquilidad y respeto durante el proceso. Es importante destacar que un factor clave que impacta los resultados es la relación terapéutica que se desarrolla entre la mujer y la enfermera (39-40).

Es necesario recalcar que las enfermeras están en la capacidad de evaluar la salud mental de las mujeres durante el control prenatal, razón por la cual deben tener el conocimiento suficiente para realizar esta intervención; además, juegan un papel importante en la detección de la ansiedad durante la atención. Se recomienda que a todas las mujeres embarazadas se les realice un examen de detección de ansiedad, para lo cual se sugiere considerar la aplicación de la Escala de trastorno de ansie- dad generalizada versión 2 (41).

A continuación, se mencionan algunos de los beneficios que reciben las gestantes antes, durante y después del parto por la asistencia de las enfermeras:

Beneficios durante el embarazo: Una de las razones por las cuales en la actualidad las gestantes que conocen los beneficios de la asistencia por parte de las enfermeras consideran la posibilidad de ser atendidas por ellas; porque saben que estas profesionales no sólo cuentan con las habilidades de atenderlas en el parto, sino que también les brindan privacidad y libertad sobre sus derechos, que en un modelo de parto obstétrico les puede ser vulnerados (32). Adicionalmente, se les brinda una asesoría integral durante el embarazo sobre autocuidado y manejo de la ansiedad; estas prácticas no sólo involucran a la futura madre y sus emociones; sino que también se tiene en cuenta a su familia y al entorno que las rodea (42-43).

Beneficios durante el parto: Las mujeres tienen una mayor sensación de confianza, lo cual trae resultados positivos en la duración, reducción del consumo de analgésicos y en la disminución de la tasa de cesáreas (44). Las enfermeras se muestran empáticas y comprensivas, permitiendo que la gestante adopte la posición en la cual se sienta más cómoda, empoderada como protagonista, confiada en su cuerpo y en su capacidad natural de dar a luz (45). En general, las enfermeras facilitan buenas prácticas en el parto, un uso menos frecuente de Oxitocina, tasas más bajas de ruptura de membranas y episiotomía, y un mayor alivio del dolor no farmacológico durante el proceso (46).

Culminado el proceso del parto: La madre ha culminado un largo proceso pero comenzará uno nuevo con un recién nacido en sus brazos; se hará cargo de todo lo relacionado con el nuevo bebé; sin embargo, la enfermera tiene un papel fundamental en el postparto ya que su acompañamiento favorecerá una menor estancia en el hospital (47). Ante esta situación, la madre se sentirá apoyada durante el complejo proceso de lactancia, ya sea materna o artificial, lo que permitirá, además, una relación única entre la madre, el recién nacido y la pareja (43-48).

Temática 2: Competencias e intervenciones de la enfermera en la atención integral de la mujer gestante.

Los partos atendidos por las enfermeras especializadas se han caracterizado por su ayuda durante la recuperación puerperal de manera natural, respetando la fisiología del cuerpo femenino; del mismo modo se evita, por ejemplo, la práctica de la episiotomía a menos de 
que sea necesaria. Por tanto, brindan un parto en donde principalmente se garantiza el acompañamiento, el manejo del dolor y una adecuada atención. $(37,48)$

Teniendo en cuenta lo anterior, es importante insistir en que la enfermera cuente con los suficientes conocimientos y una preparación integral respecto al cuidado prenatal, cuidados durante el parto y cuidados postnatales, tanto con respecto a la madre y al neonato; también, debe tener conocimientos dietéticos para mantener una buena alimentación durante el embarazo. De igual forma, es la profesional encargada de la entrega de la placenta y del manejo del cordón; además dispondrá de la capacidad de atender cualquier tipo de complicación materna, perinatal y neonatal. Esto, hará del profesional de enfermería un colaborador ideal para poder cuidar a la madre y al neonato tanto en su integridad biológica, psicológica, espiritual y social $(25,37)$.

Por otra parte, la enfermera independientemente de su grado académico, debe tener competencias para brindar apoyo emocional durante la gestación y el parto, interpretar del dolor durante el mismo, mantener una adecuada comunicación con la materna antes, durante y después de su embarazo; de igual manera estará capacitada para realizar actividades que tranquilicen a la madre durante el proceso de parto y le alivien el dolor $(24,30,34,38-40)$. Cabe resaltar, que la atención por parte de la enfermera debe ser llevada a cabo junto con la colaboración de médicos y miembros del equipo, de manera que sea una atención segura y efectiva para reducir la mortalidad materno-neonatal $(36,50)$.

\section{Cuidados e intervenciones prenatales de la enfer-} mera

Inicialmente es importante incentivar la asistencia de las gestantes a los controles prenatales que en algunos países son realizados por parte del profesional de enfermería de práctica avanzada, porque pueden detectar de manera oportuna complicaciones como malformaciones, anemia, entre otras. De igual manera, hay que realizar una valoración del riesgo materno, formular micronutrientes, garantizar el cumplimiento del esquema de vacunación y orientar acerca de los signos de alarma (24,30-31-33,35,51). También, es importante que la enfermera de práctica avanzada cuente con la capacidad de generar intervenciones orientadas a:

- Controlar el distrés respiratorio: En Colombia, esta patología presenta una incidencia que varía de 2,2 a 9,5 casos por 100.000 personas, con reportes de mortalidad que van del 30\% al 40\%. Las intervenciones del enfermero/a deben orientarse hacia el mantenimiento de una vía aérea permeable, mon- itorizar signos vitales, colocar a la paciente en una posición semifowler, iniciar el suplemento del oxígeno y auscultar ruidos respiratorios, entre otros.

- Controlar el riesgo de infección: En Colombia, las infecciones durante el embarazo, tienen una prevalencia del $11.2 \%$, siendo una de las primeras causas de muerte prenatal y neonatal tardía. Desde este punto de vista, es importante fomentar un tipo de educación que explique en qué consiste el correcto lavado de manos para la paciente, su familia y el personal de salud. Se debe fomentar el reposo, administrar un tratamiento antibiótico según la prescripción médica e instruir a la paciente y a su familia acerca de los signos y síntomas de infección, por los que deberían acudir al centro de atención.

- Controlar el riesgo de sufrir mayores alteraciones o complicaciones materno-fetales a causa de un parto prematuro: A nivel mundial 1 de cada 10 nacidos vivos son prematuros, y en Colombia su porcentaje alcanza el $7 \%$ y hasta el $12 \%$, causando morbimortalidad en el $80 \%$ de los casos; por este motivo se debe ofrecer una adecuada orientación a la gestante acerca de las posibles intervenciones que se podrían llevar a cabo durante el proceso del parto; también hay que informar sobre los riesgos fetales asociados con partos prematuros dependiendo de la edad gestacional (54).

- Control de preeclampsia severa: La preeclampsia es la manifestación hipertensiva más común en el embarazo, su frecuencia varía entre el $2 \%$ y $8 \%$, con una alta carga de morbilidad y mortalidad materna y perinatal. Una vez diagnosticada, se debe administrar oxígeno suplementario, iniciar la aplicación de sulfato de magnesio según protocolo, monitorizar reflejos osteotendinosos, control de TA y toma de exámenes de laboratorio (Hemograma, función hepática y renal).

- Control de eclampsia: La eclampsia afecta del 3\% al $22 \%$ de las mujeres embarazadas y hace parte en un $12 \%$ de las principales causas de muerte materna en los países en vías de desarrollo. Ante esta situación debe primar la intervención inmediata por parte del profesional; el/la enfermera/o debe vigilar los signos vitales, evaluar la diuresis horaria, garantizar ABC y administrar una mezcla de sulfato de magnesio.

- Control de sepsis: En Colombia, la sepsis relacionada con el embarazo tiene una prevalencia que representa el $8.9 \%$ y es considerada como una de las principales causas de muerte materna. 
Revista

Cienciagocuidado

Scientific Journal of Nursing

- Cuando se presenta la sepsis las intervenciones están encaminadas al aseguramiento de la vía aérea, administración de suplemento de oxígeno, colocar sonda vesical, iniciar manejo con líquidos IV, aplicación de antibióticos según institución y toma de paraclínicos.

Al mismo tiempo, la enfermera de práctica avanzada debe realizar un adecuado examen físico a la gestante, en donde aplique técnicas como las maniobras de Leopold Zweifel para identificar aspectos importantes del embarazo relacionados con la salud del bebé. Sin embargo, se debe tener en cuenta que es a partir del segundo trimestre del embarazo que se podrá realizar la palpación abdominal para identificar la posición y presentación fetal (27-32).

Cuidados e intervenciones de la enfermera durante el parto

Dentro de los cuidados más importantes durante el proceso del parto se encuentra el alivio del dolor, teniendo en cuenta que para minimizarlo no sólo se requiere de elementos farmacológicos, sino que también hay que proporcionar un ambiente tranquilo, en donde se atenúe la luz, se baje el tono de voz y se personalice cada parto según las necesidades $(27,30,35,49)$. Durante su atención, es importante que la enfermera conozca la cultura de la paciente, ya que le debe brindar los cuidados siguiendo sus creencias y prácticas según el tipo de población (44).

Además, las enfermeras obstétricas han utilizado diversas tecnologías de cuidado, para brindar comodidad al momento de realizar las intervenciones, que no solo beneficien a las madres, sino que también son fundamentales para el desarrollo futuro y la salud del recién nacido (52). Asimismo, las profesionales de enfermería y enfermeras de práctica avanzada no deben dejar de lado la privacidad y la autonomía de la mujer durante el proceso, alentándola a decidir acerca de sus movimientos, posiciones, comidas y otras preferencias. También, deben brindarles seguridad durante el parto, con especial atención a mujeres con obesidad o sobrepeso, ya que ellas suelen ser muy inseguras $(32,38)$.

\section{Cuidados e intervenciones postparto de la enfermera}

Es importante tener en cuenta que el periodo más crítico de la lactancia materna son las primeras dos semanas posteriores al nacimiento, por lo que se deben realizar intervenciones en donde se le enseñe a la madre a amamantar, así como a evitar la falta de leche, el peso insuficiente del bebé o pezones agrietados (29). Adi- cional a esto, las matronas deben realizar actividades que sirvan para disminuir o controlar complicaciones como profilaxis de enfermedades tromboembólicas, estimulando la deambulación temprana y el uso de medias compresivas. De igual manera, les corresponde educar a la madre acerca de los cuidados que debe tener durante el puerperio, de tal forma que pueda identificar adecuadamente los signos y síntomas de alarma por los que debería acudir a urgencias (42). Otro factor a considerar, es que la enfermera de práctica avanzada estimule y enseñe un adecuado cuidado de la salud oral, tanto de la madre como del niño a partir del primer año de edad (53).

\section{Temática 3: Consideraciones de riesgo en la asisten- cia de la gestación y el parto por enfermeras}

Conviene subrayar que las complicaciones pueden presentarse durante el embarazo, parto y postparto ya sea si este es asistido por personal de medicina o de enfermería; es por esto, que sea necesario tener en cuenta las consideraciones de riesgo para prevenirlas y así disminuir los casos de mortalidad materna (31). Sin embargo, la probabilidad de las complicaciones aumenta con factores relacionados con la falta de insumos y/o procedimientos tales como la analgesia epidural o la monitorización fetal, aspectos relacionados con la mujer como: su edad, edad gestacional, condiciones de vida, patologías de base o gestacionales, entre otros (25-31). Del mismo modo, hay que señalar que el parto se atiende con base al programa creado por el Ministerio de Salud, que incluye medidas preventivas $\mathrm{y}$ acciones de educación relativas al embarazo, parto y postparto, cumpliendo así con el objetivo de asegurar la protección materno-fetal, por medio de prácticas seguras que minimicen los riesgos (32).

Por otra parte, en los estudios revisados, se confirma que la mayor complicación en el postparto es la hemorragia, producida por las múltiples intervenciones que se realizan en el ámbito intrahospitalario y que pueden llegar a ser innecesarias. Al respecto, son causantes de la hemorragia el tacto vaginal o el uso de aparatos obstétricos que conllevan al daño de las paredes del canal vaginal, haciendo que se produzca sangrado $u$ otro tipo de complicaciones severas que pongan en riesgo la vida de la gestante o del producto de la gestación. Desde este punto de vista, es importante generar procesos articuladores entre el centro de referencia, la EPS y la secretaría de salud para la atención de emergencias, tal y como se plantea en la ruta (30). Por lo general, la hemorragia aparece cuando las pacientes han sido dadas de alta; también puede ser el resultado de la retención de restos placentarios; en este caso, se necesitaran intervenciones como el legrado y cuando es más 
grave, la histerectomía (42).

Por consiguiente, la enfermera de práctica avanzada debe contar con conocimientos científicos durante el proceso de cuidado y aplicar la taxonomía NANDA, NIC y NOC a las mujeres gestantes, teniendo en cuenta las consideraciones de riesgo descritas previamente. Algunos de los diagnósticos médicos están relacionados con riesgos: de sangrado, de infección, de alteración de la diada materno/fetal; del proceso de maternidad ineficaz; ansiedad; temor; proceso de maternidad ineficaz; $\mathrm{y}$, por último, pero no menos importante, el relacionado con el dolor de parto (57). Análogamente, las intervenciones más importantes son las que tienen que ver con: identificación de riesgos, control y detección temprana de la hemorragia (sangrado vaginal $>500 \mathrm{~mL}$ ), las cuales se deben afrontar por medio de actividades como realizar el ABC, proporcionar oxígeno suplementario, posición Trendelemburg, reanimación con cristaloides y el uso de mantas que eviten la hipotermia. Hay que precisar que estas acciones disminuyen la posibilidad de desenlaces fatales como muerte materna, perinatal o neonatal. Igualmente, se debe interpretar adecuadamente lo que tiene que ver con: útero anteparto o posparto, monitorización respiratoria, monitorización fetal electrónica y de los signos vitales antes y durante el parto. También, es importante que dentro de las intervenciones se incluya de manera transversal el manejo del dolor y del confort para la madre, sin dejar de lado el estado materno: preparto, durante y postparto. De forma adicional, no se deben olvidar los determinantes sociales de la salud de las mujeres, de acuerdo a su cultura y procedencia, puesto que son aspectos importantes para brindar una atención oportuna, según las necesidades (55).

De igual forma se identificó que en los partos de bajo riesgo asistidos por enfermeras profesionales, se presentaron menos complicaciones, puesto que no se ejecutaron prácticas de obstetricia innecesarias o invasivas, como tampoco se utilizaron fármacos como la Oxitocina (36). A nivel domiciliario hubo una disminución considerable de las dificultades en las multíparas; sin embargo, se reporta que se pueden presentar complicaciones severas y/o un mayor riesgo en el neonato de sufrir daños neurológicos o su fallecimiento. Estas situaciones se pueden evitar con prácticas seguras como: la aplicación de la estrategia de atención del minuto de oro, en donde se debe garantizar un ambiente cálido; se despeja la vía aérea en caso de ser necesario; se seca; se estimula y se reevalúa constantemente para actuar oportunamente en caso de percibir una adaptación inadecuada $(31,44)$. Por otro lado, las enfermeras mencionan que a pesar de sus esfuerzos para brindar un cuidado adecuado, podrían presentarse complicaciones en el proceso de atención al parto en el domicilio. Ante esta situación, manifiestan su incapacidad para controlar la hemorragia durante el parto y el postparto, lo que indica el traslado a la paciente a un centro asistencial, lo más pronto posible. Es por esto, que se debe hacer uso de escalas e instrumentos validados y recomendados en GPC a nivel mundial, que son de fácil aplicación en diferentes ámbitos incluyendo el domiciliario $(32-33,46)$. No obstante, Martíne-Astorquiza, afirma que, aunque el parto se lleve a cabo a nivel hospitalario puede ser un proceso fisiológico y con intervenciones mínimas; y que a su vez podrían evitarse riesgos en comparación a la asistencia domiciliaria. Adicionalmente, refiere que en países desarrollados como Holanda son muy comunes los partos en casa debido a que su sistema de salud es más ágil; pero además cuentan con servicio de ambulancia en caso de ser requerido, y que las enfermeras se encuentran capacitadas para brindar atención en el manejo de las complicaciones (55).

De acuerdo con lo planteado anteriormente, es muy importante tener en cuenta los diferentes DDS; igualmente, hay que precisar la forma como la enfermera aborda este tipo de problemas a partir del trabajo multidisciplinar, esto con el fin de prevenir complicaciones tanto en la madre como en el recién nacido y brindar una adecuada atención por medio del trabajo interinstitucional.

\section{Características de la gestante para recibir asistencia} por la enfermera.

Según las fuentes primarias consultadas, la principal característica de la gestante para ser atendida por la enfermera es que su embarazo sea de bajo riesgo; es decir, que la gestante no tenga patologías como sífilis gestacional sin tratamiento o con tratamiento interrumpido, antecedentes de malformaciones congénitas, IMC $<20$ $\mathrm{kg} / \mathrm{m} 2$, entre otros. Adicionalmente, el plan de nacimiento debe ser establecido antes de las 28 semanas de gestación, contar con cuatro controles prenatales como mínimo, en donde se indique la gestación de un solo feto y de presentación cefálica; y, por último que el trabajo de parto sea entre la semana 37 y 42 de embarazo (24-25).

Es necesario señalar que las mujeres consideradas de bajo riesgo obstétrico son aquellas que no cuentan con enfermedades de base como: diabetes, hipertensión gestacional o pregestacional, sin obesidad, VIH negativo, con un embarazo único confirmado y con parto vaginal, cefálico y a término. Además, el peso al nacer del recién nacido debe ser entre 2,500 y 4,499 gramos y los percentiles 5 a 95 para la edad gestacional (26). 
Hay que mencionar que la gestante debe tener una ganancia apropiada de peso, una ingesta de nutrientes específicos en cantidad y calidad adecuada, ya que estos factores influyen en los resultados para la madre y el recién nacido. Cabe indicar que otros rasgos como el estrés, las infecciones o hábitos tóxicos (consumo de tabaco o alcohol) pueden alterar el apetito de la embarazada, así como la disponibilidad y el metabolismo de los nutrientes, aumentando el riesgo de complicaciones perinatales (27). Es necesario insistir en que los consejos nutricionales son estrategias que deben desarrollar las enfermeras, a través de la valoración, el uso del yodo, de ácido fólico, de vitaminas y de mantener el control de peso; todo esto evita que las embarazadas consulten fuentes no confiables de información como internet o el entorno social, siendo la enfermera una fuente segura de referencia (28).

Temática 4: Retos de la enfermera en la atención durante el embarazo, parto y puerperio

Dentro de la literatura que se indagó, se describen 3 aspectos que generan retos durante el desempeño de las actividades de la enfermera obstetra. Primeramente, el profesional deberá propiciar una relación terapéutica de confianza, de tal forma que permita a las mujeres reportar toda la información necesaria para valorar y clasificar el riesgo de su gestación durante los controles prenatales, sin olvidar los determinantes sociales de la mujer. Seguidamente, se propone proveer a la mujer de herramientas que le permitan navegar dentro del sistema de salud, superando barreras que puedan retrasar el proceso de atención del binomio madre-hijo/a durante el embarazo, parto y puerperio. Por último, el trabajo interprofesional se convierte en el principal desafío, dadas las diferencias en la formación médica y de enfermería que pueden llegar a interferir en la atención. Es decir, que la profesión médica se percibe como esencialmente intervencionista frente a la de la enfermera que está más enfocada en el uso de técnicas de apoyo a las mujeres durante el trabajo de parto normal y de bajo riesgo (56). En este sentido, se propone fomentar espacios de formación que generen competencias de trabajo interprofesional desde el ámbito universitario y en diferentes campos de acción.

\section{Conclusiones}

- La presencia de la enfermera en la atención del parto permite a la gestante tener autonomía y participación en la toma de decisiones, ser respetada y comprendida sobre el proceso del parto, al proporcionarle confianza y seguridad.

- Para que las gestantes accedan a un parto con una enfermera, se recomienda realizar un proceso continuo de atención, con el fin de identificar correctamente el riesgo de la gestación. Adicionalmente las enfermeras deben estar certificadas en educación, de tal forma que les permita atender a las madres durante y después del embarazo, en las complicaciones que puedan presentarse; así como de los respectivos cuidados del postparto.

- El apoyo emocional y mental que ejerce la enfermera en la gestante es fundamental para la culminación del embarazo y para llevar a cabo el parto bajo condiciones adecuadas, factores que no sólo brindan bienestar a la futura madre y al recién nacido, sino también a su entorno familiar.

- Algunas de las gestantes optan por el parto en casa, asistido por enfermeras debido a la comodidad; esta decisión trae consigo múltiples ventajas, sobre todo en lo que tiene que ver con el trato humanizado que en muchas ocasiones no se ve reflejado en el ámbito intrahospitalario. El trabajo de la enfermera no finaliza con el nacimiento, sino que por el contrario favorece a la relación binomio madre-hijo, a la lactancia materna adecuada y al seguimiento de los signos y síntomas de las complicaciones postparto.

\section{Conflicto de Intereses}

Las autoras declaran no tener conflictos de interés.

\section{Referencias Bibliográficas}

1. Solnes MA, Roggeveen Y, Van Roosmalen J, Smith H. Factors influencing implementation of interventions to promote birth preparedness and complication readiness. Rev BMC Pregnancy Childbirth. [Internet]. 2017 [Consultado 10 de marzo de 2020]; 17(1). Disponible en: https://oi.org/10.1186/s12884-017-1448-8

2. Carvajal JA, Martinovic CP, Fernández CA. Parto Natural. ¿Qué nos dice la evidencia? Rev ARS Revista De Ciencias Médicas. [Internet]. 2017 [Consultado 10 de Marzo 2020]; 42(1). Disponible en: https://doi. org/10.11565/arsmed.v42i1.532

3. República de Colombia. Ministerio de Salud y Protección Social. Informe Quincenal Epidemiológico Nacional 
[Internet]. Instituto Nacional de Salud; El Ministerio; 2018 [Consultado 17 de marzo de 2020]; 23(13). Disponible en: https://www.ins.gov.co/buscador-eventos/IQEN/IQEN\%20vol\%2023\%202018\%20num\%2020.pdf

4. Ministerio de Salud. Guías de manejo de las complicaciones en el embarazo Programa Nacional de Salud Sexual y Reproductiva. Programa Materno Infantil. [Internet]. Panamá; 2015. [Consultado 17 de marzo de 2020]. Disponible en: http://www.minsa.gob.pa/sites/default/files/programas/guias-complicaciones-embarazo diciembre 2015.pdf

5. Ministerio De Sanidad Y Política Social. Guía de Práctica Clínica sobre la Atención al Parto Normal. [Internet]. España; 2010 [Consultado 10 de Marzo 2020];374-147 Disponible en: https://portal.guiasalud.es/wp-content/ uploads/2018/12/GPC 472_Parto_Normal_Osteba_compl.pdf

6. Programas de Naciones Unidas para el Desarrollo, Objetivos del Desarrollo Sostenible. [Internet]. 2015 [Consultado 10 de Marzo 2020];374-147 Disponible en: http://www.exteriores.gob.es/Portal/es/PoliticaExteriorCooperacion/NacionesUnidas/Paginas/ObjetivosDeDesarrolloDelMilenio.aspx

7. Organización Mundial de la Salud. Atención de enfermería y partería. [Internet] 2018. [Consultado 8 de Julio de 2020]. Disponible en: https://www.who.int/es/news-room/fact-sheets/detail/nursing-and-midwifery

8. Naciones Unidas. La Agenda 2030 y los Objetivos de Desarrollo Sostenible. [Internet] Colombia; 2018. [Consultado 8 de Julio de 2020]. Disponible en: https://repositorio.cepal.org/bitstream/handle/11362/40155/24/ $\underline{\text { S1801141_es.pdf }}$

9. Organización Mundial de la Salud.Recomendaciones de la OMS para los cuidados durante el parto, para una experiencia de parto positiva. [Internet] 2019. [Consultado 8 de Julio de 2020]. Disponible en: https://apps.who. int/iris/bitstream/handle/10665/272435/WHO-RHR-18.12-spa.pdf?ua=1

10. Ministerio de Salud y Protección Social - Colciencias. Guías de Práctica Clínica para la prevención, detección temprana y tratamiento de las complicaciones del embarazo, parto o puerperio [Internet] Colombia; 2013 [Consultado 17 de marzo de 2020]. Disponible en: https://unisalud.unal.edu.co/fileadmin/archivos/10-G.completa. Embarazo.Parto.2013.Julio17.pdf

11. Asociación Española de Matronas, Formación de la Matrona [Internet] España; 2020 [Consultado 17 de marzo de 2020]. Disponible en: https://aesmatronas.com/formacion/formacion-aem/\#1516032124463-6b0c1044-e3af

12. International Confederation of Midwives. Definición de Matrona. [Internet]. 2017 [Consultado 17 de marzo de 2020]. Disponible en: https://www.internationalmidwives.org/assets/files/definitions-files/2018/06/eng-definition of the midwife-2017.pdf

13. L. Morán-Peña La Enfermería de Práctica Avanzada ¿qué es? y ¿qué podría ser en América Latina. [Internet] Mexico;2017 [Consultado 17 de marzo de 2020]. Disponible en: https://www.elsevier.es/es-revista-enfermeria-universitaria-400-articulo-la-enfermeria-practica-avanzada-que-S1665706317300696

14. Cintra Nayara Ruiz, Riesco María Luiza González. Caracterización de los cursos de pregrado de Obstetricia en países de América del Sur. Interface (Botucatu) [Internet]. 2019 [cited 2020 July 03]; 23: e180505. Available from: http://www.scielo.br/scielo.php?script=sci arttext\&pid=S1414-32832019000100279\&lng=en.Epub Aug 26, 2019. http://dx.doi.org/10.1590/interface.180505.

15. República de Colombia. Ministerio de Salud. Resolución 3280 de 2018 [Internet]. Bogotá; El Ministerio; 2018 [Consultado 08 de abril de 2020]. Disponible en: https://www.minsalud.gov.co/Normatividad_Nuevo/Resoluci $\%$ C $3 \%$ B3n $\% 20$ No. $\% 203280 \% 20 \mathrm{de} \% 2020183280 . p d f$

16. República de Colombia. Ministerio de Salud y Protección Social. Lineamiento técnico y operativo de la Ruta Integral de Atención en Salud materno-perinatal [Internet]. Bogotá; El Ministerio; 2017 [Consultado 08 de Abril de 2020]. Disponible en: http://idsn.gov.co/site/web2/images/documentos/sreproductiva/2018/Lineamiento Ruta Materno Perinatal.pdf

17. Mendes K, Silveira R, Galvão C. Revisão integrativa: método de pesquisa para a incorporação de evidências na saúde e na enfermagem. Campus da USP, São Paulo, SP, Brasil. [Internet]. 2008 [Consultado 12 de Marzo de 2020] Disponible en: https://doi.org/10.1590/S0104-07072008000400018. 
18. Martin J, Torralba E, Díaz E, Lurueña E, Moreno S. Investigación secundaria: la revisión sistemática y el metaanálisis. Semergen Medicina de Familia; 9(34):11-16. [Internet]. [Consultado 12 de marzo de 2020]. 2008. Disponible en: https://oi.org/10.1016/S1138-3593(08)71839-2

19. CASPe. Programa de habilidades en lectura crítica español. plantillas de parrillas de lectura [Internet]. 2016 [Consultado 12 de marzo de 2020]. Disponible en: http://www.redcaspe.org/herramientas/instrumentos

20. STROBE. Lista de puntos esenciales que deben describirse en la publicación de estudios observacionales [Internet]. 2008 [Citado 12 de marzo de 2020]. Disponible en: https://www.strobe-statement.org/fileadmin/Strobe/ uploads/translations/STROBE_short_Spanish.pdf

21. República de Colombia. Ministerio de Salud. Resolución Número 8430 De 1993. [Internet]. Santa Fé de Bogotá; El ministerio; 1993 [Consultado 12 marzo de 2020]. Disponible en: https://www.minsalud.gov.co/sites/rid/Lists/ BibliotecaDigital/RIDE/DE/DIJ/RESOLUCION-8430-DE-1993.PDF

22. República de Colombia. Ministerio de Educación. Ley 911 de 2004 [Internet]. Bogotá; El ministerio; 2004 [Consultado 12 de marzo de 2020]. Disponible en: https://www.mineducacion.gov.co/1621/articles-105034_archivo pdf.pdf

23. República de Colombia. El Congreso de Colombia. Ley 1915 de 2018 [Internet]. Bogotá; El Congreso; 2018 [Consultado 12 de marzo de 2020]. Disponible en: https://www.funcionpublica.gov.co/eva/gestornormativo/norma.php?i=87419

24. Ortega Barreda E, Cairós Ventura L. et al. Panorámica internacional en relación a las recomendaciones, práctica clínica y legislación del parto en casa. [Internet]. 2017 [Consultado 2020 marzo de 18]; 11(1). Disponible en: http://scielo.isciii.es/scielo.php?script=sci_arttext\&pid=S1988-348X2017000100005\&lng=es.

25. Borges Damas L, Sixto Pérez A, Sánchez Machado R. Historia de las enfermeras obstétricas: importancia de sus cuidados en la atención al parto. Rev Cubana Enferm [Internet]. 2018 [Consultado 18 marzo de 2020];34 (3). Disponible en: http://www.revenfermeria.sld.cu/index.php/enf/article/view/1427

26. Lima p, Alves Cavalcante M, Melo S, Feitosa V, Teles de Oliveira Gouveia M. A vivência de adolescentes assistidas por enfermeiros obstetras durante o processo de parturição. Revista de Enfermagem. [Internet]. 2017 [Consultado 18 de marzo de 2020]; 7. Disponible en: http://dx.doi.org/10.19175/recom.v7i0.1823

27. Borges Damas, L.el all. El parto humanizado como necesidad para la atención integral a la mujer. Revista Cubana de Obstetricia y Ginecología [Internet]. 2018 [Consultado 18 de marzo de 2020] Disponible en: http:// scielo.sld.cu/pdf/gin/v44n3/gin02318.pdf

28. Lundeen T, Musange S, Azman H, Nzeyimana D, Murindahabi N. et al. Nurses' and midwives' experiences of providing group antenatal and postnatal care at 18 health centers in Rwanda: A mixed methods study. Rev Plos One [Internet]. 2019 [Consultado 18 de marzo de 2020]; 14(7). Disponible en: https://journals.plos.org/plosone/ article/file?id=10.1371/journal.pone.0219471\&type=printable

29. Bedwell C, McGowan L, Lavender D. Factors affecting midwives' confidence in intrapartum care: A phenomenological study. Rev Midwifery. [Internet]. 2015 [Consultado 18 de marzo de 2020]; 31(1). Disponible en: https:// doi.org/10.1016/j.midw.2014.08.004

30. Jurado Bleda J. El proceso del parto en el domicilio. [Tesis en internet] Escuela superior de ciencias de la salud inscrita a UPF; 2018 [Consultado 18 de marzo de 2020]. Disponible en: http://repositori.tecnocampus.cat/bitstream/handle $/ 20.500 .12367 / 60 / J u r a d o \% 20 B l e d a \% 20 J u d i t h \_E l \% 20$ proceso $\% 20$ del $\% 20$ parto $\% 20 \mathrm{en} \% 20$ domicilio.pdf?sequence $=1 \&$ isAllowed $=\mathrm{y}$

31. Marín Sánchez MP. Aspectos bioéticos del parto natural. [Tesis en internet] Universidad de Murcia; 2015 [Consultado 18 de marzo de 2020]. Disponible en: https://digitum.um.es/digitum/bitstream/10201/44754/1/ASPECTOS\%20BIO $\%$ c3\%89TICOS\%20DEL \%20PARTO\%20NATURAL.pdf

32. Capellari Fabrizzio G, Moreira Schmalfuss J, Silveira L, Cechinel Peiter C, Guedes dos Santos J, Lorenzini 
Erdmann A. Práticas obstétricas de uma parteira: contribuições para a gestão do cuidado de enfermagem à parturiente, Rev de Enfermagem do Centro-Oeste Mineiro, [İnternet]. 2019 [Consultado 18 de marzo de 2020]; 9. Disponible en: https://doi.org/10.19175/recom.v9i0.2892

33. Amutah-Onukagha N, Rodriguez M, Opara I, Gardner M, Assan M. et al. Progresses and challenges of utilizing traditional birth attendants in maternal and child health in Nigeria. Rev Int J MCH AIDS. [Internet]. 2017 [Consultado 18 de marzo de 2020]; 6(2) Disponible en: https://www.ncbi.nlm.nih.gov/pmc/articles/PMC5777388/

34. Maputle M. Support provided by midwives to women during labour in a public hospital, Limpopo Province, South Africa: a participant observation study. Rev BMC Pregnancy Childbirth. [Internet]. 2018 [Consultado 18 de marzo de 2020]; 18(1). Disponible en: https://bmcpregnancychildbirth.biomedcentral.com/articles/10.1186/ $\underline{\text { s12884-018-1860-8 }}$

35. Antunes Ramos W, Costa Aguilar B, Conrad D, Baptista Pinto C, Amaral Mussumeci P. Contribuição da enfermeira obstétrica nas boas práticas da assistência ao parto e nascimento. Rev Fund Care Online. [Internet]. 2018 [Consultado 17 de marzo de 2020]; 10(1). Disponible en: http://dx.doi.org/10.9789/2175-5361.2018.v10i1.173-179

36. Nogueira da Gama S, Fernandes Viellas E, Alves Torres J, Bastos M, Brüggemann O, et al. Labor and birth care by nurse with midwifery skills in Brazil. Rev Reprod Health. [Internet]. 2016 [Consultado 17 de marzo de 2020]; 13(3). Disponible en: https://doi.org/10.1186/s12978-016-0236-7

37. Fernández Turienzo C, Bick D, Bollard M, Brigante L, Briley A. et al. POPPIE: protocol for a randomised controlled pilot trial of continuity of midwifery care for women at increased risk of preterm birth. Rev Trials. [Internet]. 2019 [Consultado 18 de marzo de 2020]; 20(1). Disponible en: https://rialsjournal.biomedcentral. com/articles/10.1186/s13063-019-3352-1

38. Daemers D, Limbeek E, Wijnen H, Nieuwenhuijze M, Vries R. Factors influencing the clinical decision-making of midwives: a qualitative study. Rev BMC Pregnancy Childbirth. [Internet]. 2017 [Consultado 18 de marzo de 2020]. Disponible en: https://doi.org/10.1186/s12884-017-1511-5

39. Macias Intrago M, Haro Alvarado J, Piloso Gómez E, Galarza Soledispa G, Quishpe Molina M, Triviño Vera B. Importancia y beneficios del parto humanizado. Rev Dom. Cien. [Internet]. 2018 [Consultado 18 de marzo de 2020]; 4(3). Disponible en: https://dialnet.unirioja.es/servlet/articulo?codigo $=6560206$

40. Kildea S, Simcock G, Liu A, Elgbeili G, Laplante D, et al. Continuity of midwifery carer moderates the effects of prenatal maternal stress on postnatal maternal wellbeing: the Queensland flood study. Rev Arch Womens Ment Health. [Internet]. 2017 [Consultado 17 de marzo de 2020]; 21(2). Disponible en: https://www.unboundmedicine.com/medline/citation/28956168/Continuity of midwifery carer moderates the effects of prenatal_maternal_stress_on_postnatal_maternal_wellbeing: the

41. McGookin A, Furber C, Smith D. Student midwives' awareness, knowledge, and experiences of antenatal anxiety within clinical practice. Rev J Reprod Infantil Psychol. [Internet]. 2017 [Consultado 17 de marzo de 2020]; 35(4). Disponible en: https://pubmed.ncbi.nlm.nih.gov/29517371/

42. Pérez Martínez E. Relación entre la atención exclusiva de la matrona al puerperio de bajo riesgo y el número de mujeres que acuden a la urgencia de obstetricia por complicaciones posparto. [Tesis en internet]. Universidad Complutense De Madrid; 2016 [Consultado 18 de marzo de 2020] Disponible en: https://eprints.ucm.es/49813/1/ T40503.pdf

43. Espuig Sebastián R, Noreña A, Cortés Castell E, González-Sanz J. Percepción de embarazadas y matronas acerca de los consejos nutricionales durante la gestación. Rev Nutr. Hosp. [Internet]. 2016 [Consultado 17 de marzo de 2020]; 33(5). Disponible en: http://scielo.isciii.es/scielo.php?script=sci_arttext\&pid=S0212-16112016000500028\&lng=es

44. Angarita Navarro A. El acompañamiento por enfermería en la atención del trabajo de parto y parto: una revisión narrativa de la literatura. Rev Investig Salud Univ Boyacá [Internet]. 2018 [Consultado 18 de marzo de 2020]; (5)2. Disponible en: https://doi.org/10.24267/23897325.346

45. Kozhimannil K, Attanasio L, Yang T, Avery M, Declercq E. Midwifery care and patient-provider communica- 
tion in maternity decisions in the United States. Rev Matern Child Health J. [Internet]. 2015 [Consultado 18 de marzo de 2020]; 19(7). Disponible en: https://www.ncbi.nlm.nih.gov/pmc/articles/PMC4476913/

46. Thommesen T, Kismul H, Kaplan I, Safi K, Van den Bergh G. "The midwife helped me ... otherwise I could have died": women's experience of professional midwifery services in rural Afghanistan - a qualitative study in the provinces Kunar and Laghman. Rev BMC. [Internet]. 2020 [Consultado 17 de marzo de 2020]; 20(1). Disponible en: https://bmcpregnancychildbirth.biomedcentral.com/articles/10.1186/s12884-020-2818-1

47. Forster D, McLachlan H, Davey M, Biro M, Farrell T, Gold L. Continuity of care by a primary midwife (caseload midwifery) increases women's satisfaction with antenatal, intrapartum and postpartum care: results from the COSMOS randomised controlled tria. Rev BMC Pregnancy and Childbirth. [Internet]. 2016 [Consultado 17 de marzo de 2020]; 16(1). Disponible en: https://doi.org/10.1186/s12884-016-0798-y

48. Swerts M, Westhof E, Bogaerts A, Lemiengre J. Supporting breastfeeding women from the perspective of the midwife: a systematic review of the literature. Rev Midwifery. [Internet]. 2016 [Consultado 17 de marzo de 2020]; 37. Disponible en: https://doi.org/10.1016/j.midw.2016.02.016

49. Munabi-Babogumira S, Glenton C, Lewin S, Fretheim A, Nabudere H. Factors that influence the provision of intrapartum and postnatal care by skilled birth attendants in low- and middle-income countries: a qualitative evidence synthesis. Rev Cochrane Library. [Internet]. 2017 [Consultado 17 de marzo del 2020]; 11(11). Disponible en: https://www.ncbi.nlm.nih.gov/pmc/articles/PMC5721625/

50. Hildingsson I, Gamble J, Sidebotham M, Creedy D, Guilliland K, Dixon L,et al. Midwifery empowerment: National surveys of midwives from Australia, New Zealand and Sweden. Rev Midwifery. [Internet]. 2016 [Consultado 17 de marzo de 2020]; 40. Disponible en: https://doi.org/10.1016/j.midw.2016.06.008

51. Cano M, Valiente I, Reina A. Importancia de la asistencia de la matrona durante el puerperio para mejorar la adaptación a la maternidad. Rev Biblioteca Lascasas. [Internet]. 2015 [Consultado 17 de marzo de 2020]; 11(3). .Disponible en: http://www.index-f.com/lascasas/documentos/lc0841.pdf

52. Bali S, Reddy VB. Evaluation of competency and skills of skilled birth attendants in Madhya Pradesh, central India. Indian journal of public health [Internet].2018 [Consultado 30 de junio de 2020];62(1). Disponible en:10.4103/ijph.IJPH_332_16

53. Wagner Y, Heinrich-Weltzien R. Midwives' oral health recommendations for pregnant women, infants and young children: results of a nationwide survey in Germany. Rev BMC oral health. [Internet]. 2016 [Consultado 17 de marzo de 2020];16(1).Disponible en: https://doi.org/10.1186/s12903-016-0192-1

54. Romero I. Proceso De Atención De Enfermería En Gestantes Con Complicaciones Obstétricas Por Ruptura Prematura De Membranas. Rev UTMACH [Internet]. 2018 [Consultado 30 de junio de 2020]; 22. Disponible en: http://repositorio.utmachala.edu.ec/bitstream/48000/13133/1/E-8485 CONTENTO\%20ROMAN\%20ANGELA\%20JAZMIN.pdf

55. Aragón Núñez M, Cubillas Rodríguez I, García Rojas I. La bienvenida de Leo. Parto en casa. [Internet]. 2017 [Consultado 17 de marzo de 2020]; Disponible en: http:/www.huvv.es/sites/default/files/revistas/11bienvenida $\% 20 \mathrm{de} \% 201$ eo, $\% 20$ publicable_CORREGIDO $\% 20$ teresa.pdf

56. Crisóstomo M, Lemos S, Gnocchi da Costa Reis L, Martin M, Louise Aveling E. Challenges to the improvement of obstetriccare in maternity hospitals of a largeBrazilian city: an exploratory qualitativeapproach on contextual issues. Rev BMC Pregnancy Childbirth. [Internet]. 2018 [Consultado 9 de abril de 2021] Disponible en: https://doi.org/10.1186/s12884-018-2088-3

57. Bulechek G, Howard K, Dochterman J. Nnnconsult[Internet]; Elsevier. 2020 [Consultado 26 julio de 2020]. Disponible en: https://www.nnnconsult.com/ 